\title{
Time-reversal invariant and fully gapped unconventional superconducting state in the bulk of the topological compound $\mathrm{Nb}_{0.25} \mathrm{Bi}_{2} \mathrm{Se}_{3}$
}

\author{
Debarchan Das $\odot,{ }^{1}$ K. Kobayashi $\odot,{ }^{2}$ M. P. Smylie $\odot,{ }^{3}$ C. Mielke III $\odot,{ }^{1}$ T. Takahashi, ${ }^{2}$ K. Willa $\odot,{ }^{4}$ J.-X. Yin $\odot,{ }^{5}$ U. Welp, ${ }^{6}$ \\ M. Z. Hasan, ${ }^{5}$ A. Amato, ${ }^{1}$ H. Luetkens $\odot,{ }^{1}$ and Z. Guguchia $\oplus^{1, *}$ \\ ${ }^{1}$ Laboratory for Muon Spin Spectroscopy, Paul Scherrer Institute, CH-5232 Villigen PSI, Switzerland \\ ${ }^{2}$ Research Institute for Interdisciplinary Science, Okayama University, Okayama 700-8530, Japan \\ ${ }^{3}$ Department of Physics and Astronomy, Hofstra University, Hempstead, New York 11549, USA \\ ${ }^{4}$ Institute for Solid State Physics, Karlsruhe Institute of Technology, Karlsruhe D-76021, Germany \\ ${ }^{5}$ Laboratory for Topological Quantum Matter and Spectroscopy, Department of Physics, Princeton University, \\ Princeton, New Jersey 08544, USA \\ ${ }^{6}$ Materials Science Division, Argonne National Laboratory, 9700 South Cass Avenue, Lemont, Illinois 60439, USA
}

(Received 26 August 2020; revised 5 October 2020; accepted 6 October 2020; published 29 October 2020)

\begin{abstract}
Recently, the niobium $(\mathrm{Nb})$ doped topological insulator $\mathrm{Bi}_{2} \mathrm{Se}_{3}$, in which the finite magnetic moments of the $\mathrm{Nb}$ atoms are intercalated in the van der Waals gap between the $\mathrm{Bi}_{2} \mathrm{Se}_{3}$ layers, has been shown to exhibit both superconductivity with $T_{c} \simeq 3 \mathrm{~K}$ and topological surface states. Here we report on muon spin rotation experiments probing the temperature and field dependence of effective magnetic penetration depth $\lambda_{\text {eff }}(T)$ in the layered topological superconductor candidate $\mathrm{Nb}_{0.25} \mathrm{Bi}_{2} \mathrm{Se}_{3}$. The exponential temperature dependence of $\lambda_{\text {eff }}^{-2}(T)$ at low temperatures suggests a fully gapped superconducting state in the bulk with the superconducting transition temperature $T_{c}=2.9 \mathrm{~K}$ and the gap to $T_{c}$ ratio $2 \Delta / k_{B} T_{c}=3.95(19)$. We also reveal that the ratio $T_{c} / \lambda_{\text {eff }}^{-2}$ is comparable to those of unconventional superconductors, which hints at an unconventional pairing mechanism. Furthermore, time-reversal symmetry breaking was excluded in the superconducting state with sensitive zero-field $\mu \mathrm{SR}$ experiments. We hope the present results will stimulate theoretical investigations to obtain a microscopic understanding of the relation between superconductivity and the topologically nontrivial electronic structure of $\mathrm{Nb}_{0.25} \mathrm{Bi}_{2} \mathrm{Se}_{3}$.
\end{abstract}

DOI: 10.1103/PhysRevB.102.134514

\section{INTRODUCTION}

Topological superfluidity and superconductivity are wellestablished phenomena in condensed matter systems. A very good example of a topological system is the $A$ phase of superfluid helium-3 [1], which is a charge-neutral topological superfluid. Topological superconductors (TSCs) are special families of those materials with unique electronic states, a full pairing gap in the bulk, and gapless surface states consisting of Majorana fermions (MFs) [2-6]. Due to their scientific importance and potential applications in quantum computing, MFs have attracted much attention recently [7-9]. This stimulating aspect of TSCs motivated the condensed matter community to design novel topological systems by inducing superconductivity in a material with a nontrivial topological band structure. Ways to realize TSCs include through the proximity at the interface between $s$-wave superconductors and topological insulators (TIs) with large spin-orbit coupling [9], at superconductor-magnet interfaces (1D chiral Majorana edge states), and at SC-TI-SC Josephson junctions (1D helical Majorana edge states). Another pathway to design TSCs is to induce superconductivity in the bulk of TIs by doping, intercalation, or pressure [10-14]. Currently, there are a few

*zurab.guguchia@psi.ch potential material candidates for bulk TSCs, obtained by following the latter approach: $\mathrm{Sr}_{2} \mathrm{RuO}_{4}$ [15], which is generally believed to be a topological time-reversal symmetry (TRS) breaking superconductor, the Weyl semimetal $T_{d}-\mathrm{MoTe}_{2}$ [16], which is reported to be a TRS-invariant topological superconductor, and $\mathrm{Cu} / \mathrm{Sr} / \mathrm{Nb}$-doped $\mathrm{Bi}_{2} \mathrm{Se}_{3}$ created from one of the most studied TIs, $\mathrm{Bi}_{2} \mathrm{Se}_{3}$ [17-24], which is reported to be a nematic [25] superconductor. The distinguishing feature of the $\mathrm{Bi}_{2} \mathrm{Se}_{3}$-derived superconductors is the emergence of a pronounced twofold in-plane anisotropy of all superconducting properties [26] even though the crystal structure has threefold symmetry [Fig. 1(a)]. This so-called nematic behavior was ascribed to an unconventional odd-parity twocomponent superconducting order parameter of $E_{u}$ symmetry which preserves time-reversal symmetry [14,25,27,28]. The two components, $\Delta_{4 x}$ and $\Delta_{4 y}$, are characterized by two inplane point nodes and deep minima, respectively, defining the nematic axis.

In this framework, the $\mathrm{Nb}$-doped $\mathrm{Bi}_{2} \mathrm{Se}_{3}$ system appears to be unique because macroscopic magnetic ordering below the superconducting critical temperature of $3.2 \mathrm{~K}$ has been reported [29], indicating a spontaneous spin rotation symmetry breaking of the $\mathrm{Nb}$ magnetic moments and TRS breaking. Nevertheless, detailed experimental studies involving magnetoresistance, surface-sensitive penetration depth measurements, and Andreev reflection spectroscopy 

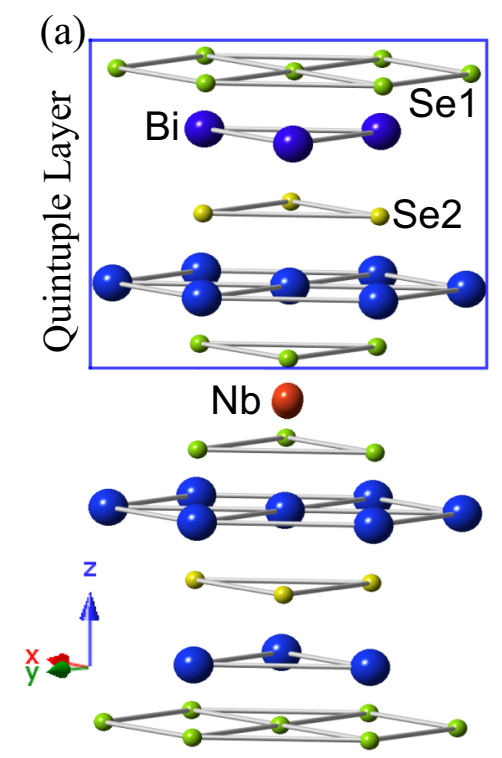

(b)

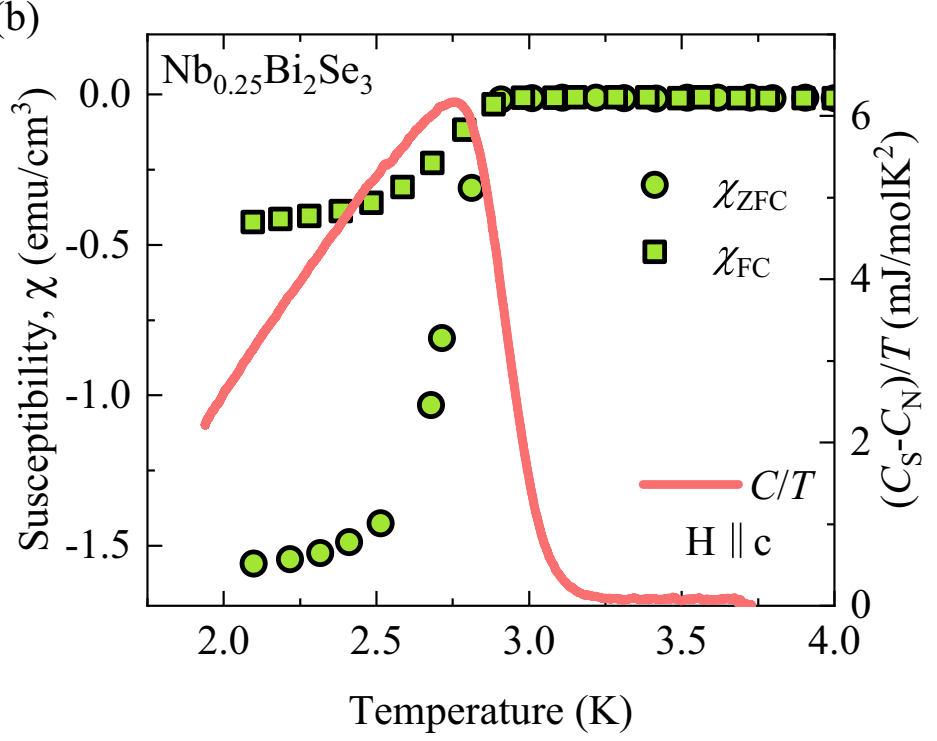

FIG. 1. Crystal structure and bulk superconducting transition for $\mathrm{Nb}_{0.25} \mathrm{Bi}_{2} \mathrm{Se}_{3}$. (a) Crystal structure of $\mathrm{Nb}$-doped $\mathrm{Bi}_{2} \mathrm{Se}_{3}$ emphasizing the position of $\mathrm{Nb}$ intercalation between $\mathrm{Bi}_{2} \mathrm{Se}_{3}$ layers (highlighted as quintuple layer). (b) Temperature dependence of DC magnetic susceptibility (left axis) in $H(\| c)=1 \mathrm{mT}$ and zero-field superconducting part of the specific heat divided by temperature (right axis) for the single-crystalline sample of $\mathrm{Nb}_{0.25} \mathrm{Bi}_{2} \mathrm{Se}_{3}$. The susceptibility and specific-heat measurements show similar transition temperature $(\sim 2.9 \mathrm{~K})$.

corroborate the existence of a nematic phase in the $\mathrm{Nb}$-doped $\mathrm{Bi}_{2} \mathrm{Se}_{3}$ system [21,22,30-32]. Due to the relatively large superconducting volume fraction in $\mathrm{Nb}$-doped $\mathrm{Bi}_{2} \mathrm{Se}_{3}$ compared to its $\mathrm{Cu}$ counterpart, it can be an ideal material to investigate the superconducting properties in detail enabling further insight into the physics of TSCs. However, there are limited experimental studies reported exploring the bulk superconducting properties of this system. Thus, thorough exploration of superconductivity on the microscopic level in the bulk of $\mathrm{Nb}_{0.25} \mathrm{Bi}_{2} \mathrm{Se}_{3}$ from both experimental and theoretical perspectives is required. In this regard, we concentrate on muon spin rotation/relaxation/resonance ( $\mu \mathrm{SR})$ measurements of the magnetic penetration depth $\lambda$ in $\mathrm{Nb}_{0.25} \mathrm{Bi}_{2} \mathrm{Se}_{3}$, which is one of the fundamental parameters of a superconductor, since it is related to the superfluid density $n_{s}$ via $1 / \lambda^{2}=\mu_{0} e^{2} n_{s} / m^{*}$ (where $m^{*}$ is the effective mass). Most importantly, the temperature dependence of $\lambda$ is particularly sensitive to the structure of the SC gap. Moreover, zero-field $\mu$ SR is a very powerful tool for detecting a spontaneous magnetic field due to TRS breaking in exotic superconductors, because internal magnetic fields as small as $0.1 \mathrm{G}$ are detected in measurements without applying external magnetic fields.

We report on fully gapped and a time-reversal invariant superconducting state in the bulk of topological system $\mathrm{Nb}_{0.25} \mathrm{Bi}_{2} \mathrm{Se}_{3}$. We evaluated the higher limit of the zero-temperature penetration depth to be $\sim 930(10) \mathrm{nm}$. Interestingly, the $T_{c} / \lambda_{\text {eff }}^{-2}$ ratio is comparable to those of unconventional superconductors. The relatively high $T_{c}$ for small carrier density may hint at an unconventional pairing mechanism in $\mathrm{Nb}_{0.25} \mathrm{Bi}_{2} \mathrm{Se}_{3}$.

\section{EXPERIMENTAL DETAILS}

The details of the synthesis of the single-crystal samples of $\mathrm{Nb}_{0.25} \mathrm{Bi}_{2} \mathrm{Se}_{3}$ are reported elsewhere [23]. High-purity sam- ples were produced by properly selecting the crystals from the melt. Magnetization, x-ray diffraction, and specific-heat experiments were performed on the single crystals, while $\mu$ SR experiments were measured on powdered crystals from the same batch. X-ray diffraction analysis confirmed that the synthesized samples are single-phase materials. There is no trace of $\mathrm{BiNbSe}_{3}$ phase in our sample. The magnetization measurements were performed in a commercial SQUID magnetometer (Quantum Design MPMS-XL). AC specific heat was measured on a $\mathrm{SiN}$ membrane-based nanocalorimeter platform [33]. The temperature dependence of the superconducting contribution [34] (the normal-state electronic specific heat is subtracted) was extracted by subtracting data with $H(\| c)=1 \mathrm{~T}$ from the zero-field data [35]. The specific heat was scaled to match the published data [32] in the normal state as a precise determination of the sample volume was not possible due to the small mass of the sample.

Transverse-field (TF) and zero-field (ZF) $\mu$ SR experiments were performed at the $\pi \mathrm{E} 1$ beamline of the Paul Scherrer Institute (Villigen, Switzerland), using the Dolly spectrometer. The sample was pressed into a $5 \mathrm{~mm}$ pellet and mounted on $\mathrm{a} \mathrm{Cu}$ holder using GE varnish. This holder assembly was then fixed to the ${ }^{3} \mathrm{He}$ cryostat. The spectrometer is equipped with a standard veto setup providing a low-background $\mu$ SR signal. All TF experiments were carried out after a field-cooling procedure in the temperature range of $0.26-5 \mathrm{~K}$. The $\mu \mathrm{SR}$ time spectra were analyzed using the free software package MUSRFIT [36].

\section{RESULTS}

Figure 1(a) shows the anisotropic layered rhombohedral crystal structure of $\mathrm{Nb}$-intercalated $\mathrm{Bi}_{2} \mathrm{Se}_{3}$. $\mathrm{Nb}$ atoms are residing in the van der Waals gap between adjacent $\mathrm{Bi}_{2} \mathrm{Se}_{3}$ quintuple $\mathrm{Se}-\mathrm{Bi}-\mathrm{Se}-\mathrm{Bi}-\mathrm{Se}$ layers (five covalently bonded 

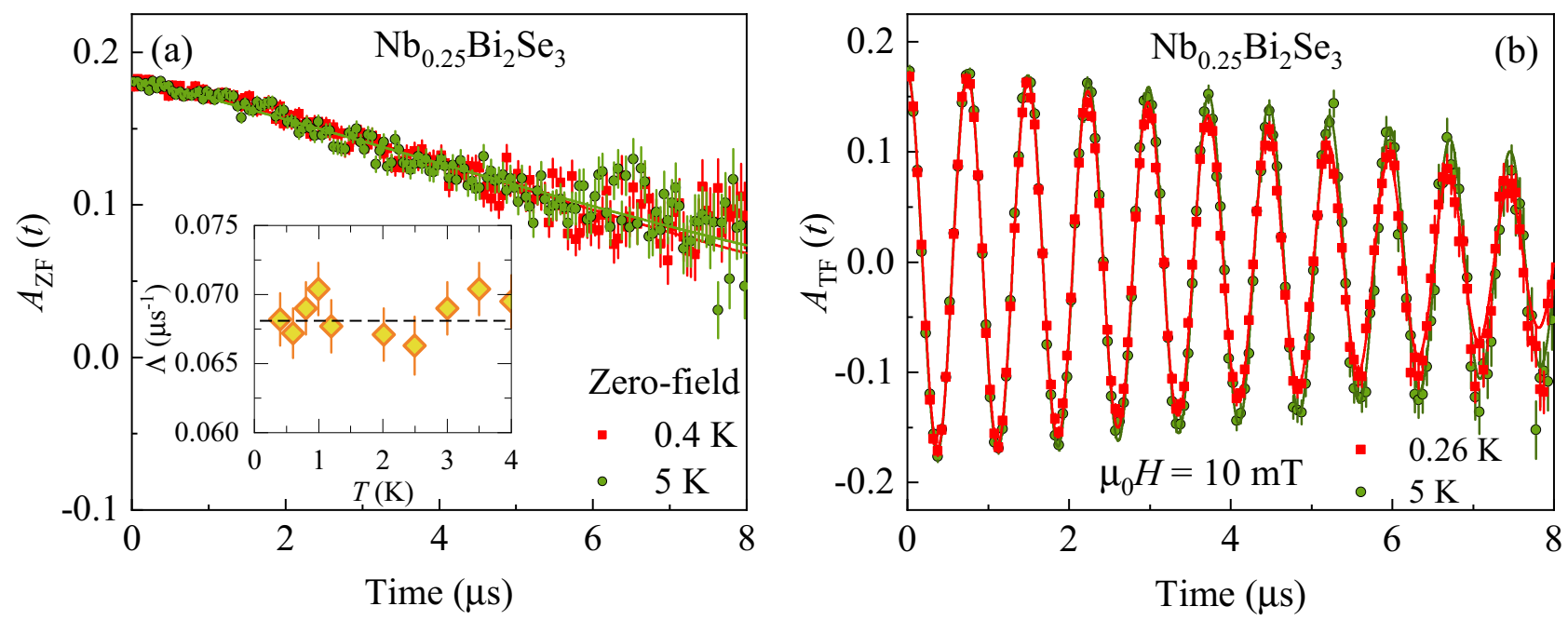

FIG. 2. Zero-field (ZF) and transverse-field (TF) $\mu \mathrm{SR}$ time spectra for $\mathrm{Nb}_{0.25} \mathrm{Bi}_{2} \mathrm{Se}_{3}$. Zero-field $\mu \mathrm{SR}$ (a) and transverse-field (b) $\mu \mathrm{SR}$ time spectra obtained above and below $T_{c}$ for $\mathrm{Nb}_{0.25} \mathrm{Bi}_{2} \mathrm{Se}_{3}$ (after field-cooling the sample from above $T_{c}$ ). Inset of (a) shows the temperature dependence of the electronic relaxation rate measured in zero magnetic field of $\mathrm{Nb}_{0.25} \mathrm{Bi}_{2} \mathrm{Se}_{3}$ with $T_{c} \sim 3 \mathrm{~K}$. Error bars are the standard error of the mean in about $10^{6}$ events. The error of each bin count $n$ is given by the standard deviation of $n$. The errors of each bin in $A(t)$ are then calculated by standard-error propagation.

atomic sheets) as shown in the figure. The quintuple layers are weakly bonded together by van der Waals interaction. The left axis of Fig. 1(b) shows the temperature dependence of field-cooled (FC) and zero-field-cooled (ZFC) magnetic susceptibility $\chi(T)$ for the single crystal in an applied magnetic field of $H(\| c)=1 \mathrm{mT}$. A clear diamagnetic transition appears in both FC and ZFC plots with a critical temperature $T_{c} \simeq 2.9 \mathrm{~K}$ and with $100 \%$ superconducting volume fraction, confirming the onset of bulk superconductivity in studied $\mathrm{Nb}_{0.25} \mathrm{Bi}_{2} \mathrm{Se}_{3}$. To elucidate the intrinsic and bulk nature of this superconducting phase transition, we measured specific heat on a single-crystalline sample of $\mathrm{Nb}_{0.25} \mathrm{Bi}_{2} \mathrm{Se}_{3}$. The right axis of Fig. 1(b) shows the temperature variation of the superconducting contribution to the specific heat divided by the temperature which exhibits a prominent steplike anomaly at $T_{c}$, indicating bulk superconductivity in this system.

First, we have carried out ZF- $\mu$ SR experiments above and below $T_{c}$ to search for possible magnetism (static or fluctuating) in $\mathrm{Nb}_{0.25} \mathrm{Bi}_{2} \mathrm{Se}_{3}$. As shown in Fig. 2(a), no sign of either static or fluctuating magnetism could be detected in $\mathrm{ZF}$ time spectra down to $0.4 \mathrm{~K}$. The $\mathrm{ZF}-\mu \mathrm{SR}$ spectra can be well described by a damped Gaussian Kubo-Toyabe depolarization function [37], reflecting the field distribution at the muon site created by the nuclear moments. Moreover, no change in ZF- $\mu$ SR relaxation rate across $T_{c}$ was observed [see inset of Fig. 2(a)], pointing to the absence of any spontaneous magnetic fields associated with a TRS [15,38] breaking pairing state in $\mathrm{Nb}_{0.25} \mathrm{Bi}_{2} \mathrm{Se}_{3}$. This demonstrates that there is, in fact, no magnetic ordering in $\mathrm{Nb}_{0.25} \mathrm{Bi}_{2} \mathrm{Se}_{3}$.

Figure 2(b) represents the $\mathrm{TF}-\mu \mathrm{SR}$ spectra for $\mathrm{Nb}_{0.25} \mathrm{Bi}_{2} \mathrm{Se}_{3}$, measured in an applied magnetic field of $10 \mathrm{mT}$ above $(5 \mathrm{~K})$ and below $(0.26 \mathrm{~K})$ the $\mathrm{SC}$ transition temperature $T_{c}$. Above $T_{c}$ the oscillations show a small relaxation due to the random local fields from the nuclear magnetic moments. At $0.26 \mathrm{~K}$, the relaxation rate increases due to the presence of a nonuniform local field distribution as a result of the formation of a flux-line lattice (FLL) in the SC state. As indicated by solid lines in Fig. 2(a), TF- $\mu$ SR data were analyzed by using the following functional form [36]:

$$
A_{\mathrm{TF}_{S}}(t)=A_{S} e^{\left[-\frac{\left(\sigma_{\mathrm{sc}}^{2}+\sigma_{\mathrm{nm}}^{2}\right) t^{2}}{2}\right]} \cos \left(\gamma_{\mu} B_{\mathrm{int}} t+\varphi\right) .
$$

Here $A_{S}$ denotes the initial asymmetry, $\gamma_{\mu} /(2 \pi) \simeq 135.5$ $\mathrm{MHz} / \mathrm{T}$ is the muon gyromagnetic ratio, and $\varphi$ is the initial phase of the muon-spin ensemble. $B_{\text {int }}$ represents the internal magnetic field at the muon site; the relaxation rates $\sigma_{\mathrm{sc}}$ and $\sigma_{\mathrm{nm}}$ characterize the damping due to the formation of the FLL in the SC state and of the nuclear magnetic dipolar contribution, respectively. During the analysis $\sigma_{\mathrm{nm}}$ was assumed to be constant over the entire temperature range and was fixed to the value obtained above $T_{c}$ where only nuclear magnetic moments contribute to the muon depolarization rate $\sigma$.

In Fig. 3(a), $\sigma_{\mathrm{sc}}$ is plotted as a function of temperature for $\mathrm{Nb}_{0.25} \mathrm{Bi}_{2} \mathrm{Se}_{3}$ at $\mu_{0} H=0.01 \mathrm{~T}$. Below $T_{c}$ the relaxation rate $\sigma_{\mathrm{sc}}$ starts to increase from zero due to the formation of the FLL and shows the saturation toward the low temperatures. We show in the following paragraph that the observed temperature dependence of $\sigma_{\mathrm{sc}}$, which reflects the topology of the SC gap, is consistent with the presence of the single SC gap on the Fermi surface of $\mathrm{Nb}_{0.25} \mathrm{Bi}_{2} \mathrm{Se}_{3}$. It is interesting to note that below $T_{c}$, we observed a paramagnetic shift of the internal magnetic field $B_{\text {int }}$, sensed by the muons, instead of the expected diamagnetic shift imposed by the SC state. This is evident in Fig. 3(b), where we plot the difference between the internal field $B_{\text {int,SC }}$ measured in the SC state and one $B_{\text {int,NS }}$ measured in the normal state $T=5 \mathrm{~K}$.

To proceed with a quantitative analysis, we note that the temperature dependence of the London magnetic penetration depth $\lambda(T)$ is related to the muon spin depolarization rate $\sigma_{\mathrm{sc}}(T)$ in the presence of a perfect triangular vortex lattice by the equation $[39,40]$

$$
\frac{\sigma_{\mathrm{sc}}^{2}(T)}{\gamma_{\mu}^{2}}=0.00371 \frac{\Phi_{0}^{2}}{\lambda^{4}(T)},
$$



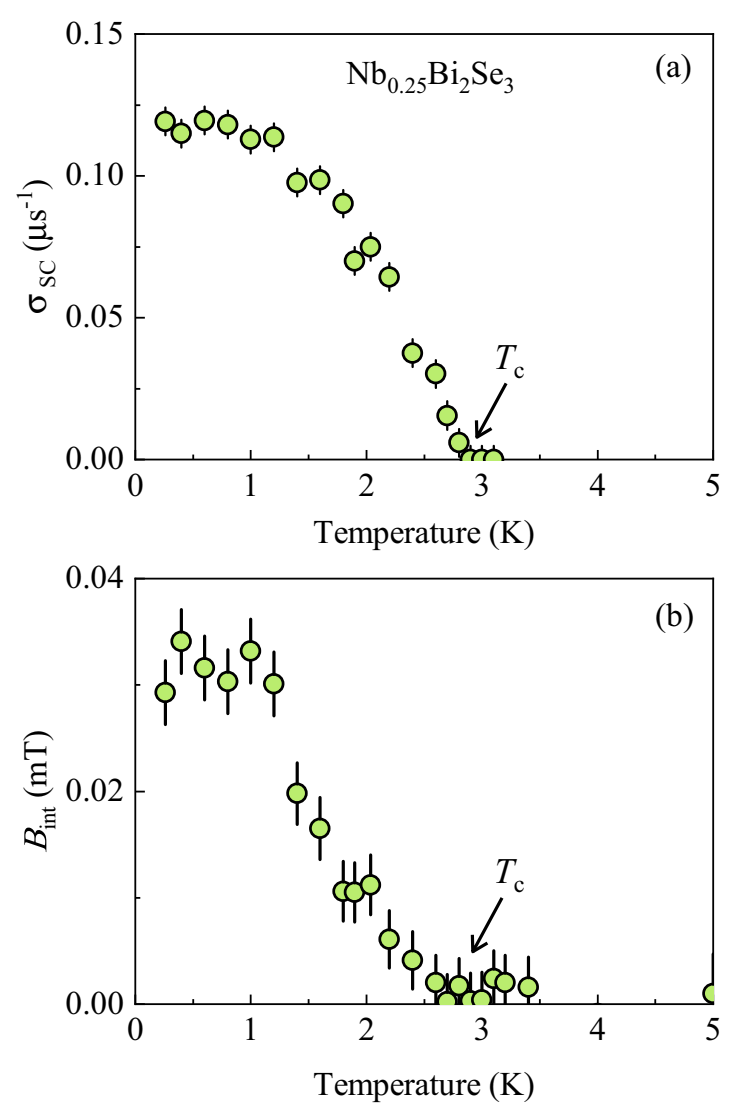

FIG. 3. Superconducting muon spin depolarization rate $\sigma_{\mathrm{sc}}$ and the field shift for $\mathrm{Nb}_{0.25} \mathrm{Bi}_{2} \mathrm{Se}_{3}$. (a) Temperature dependence of the superconducting muon spin depolarization rate $\sigma_{\mathrm{sc}}$ measured in an applied magnetic field of $\mu_{0} H=0.01 \mathrm{~T}$ for $\mathrm{Nb}_{0.25} \mathrm{Bi}_{2} \mathrm{Se}_{3}$. The error bars represent the standard deviation of the fit parameters. (b) Temperature dependence of the difference between the internal field $\mu_{0} H_{\mathrm{SC}}$ measured in the SC state and the one measured in the normal state $\mu_{0} H_{\mathrm{NS}}$ at $T=5 \mathrm{~K}$ for $\mathrm{Nb}_{0.25} \mathrm{Bi}_{2} \mathrm{Se}_{3}$.

where $\Phi_{0}=2.068 \times 10^{-15} \mathrm{~Wb}$ is the magnetic-flux quantum. Equation (2) is only valid when the separation between the vortices is smaller than $\lambda$. In this case, according to the London model, $\sigma_{\mathrm{sc}}$ is field independent [39].

To investigate the superconducting gap structure of $\mathrm{Nb}_{0.25} \mathrm{Bi}_{2} \mathrm{Se}_{3}$, we analyzed the $T$ dependence of the magnetic penetration depth, $\lambda(T)$, which is directly associated with the superconducting gap. $\lambda(T)$ can be described within the local (London) approximation $(\lambda \gg \xi)$ by the following expression $[36,41]$ :

$$
\frac{\lambda^{-2}\left(T, \Delta_{0, i}\right)}{\lambda^{-2}\left(0, \Delta_{0, i}\right)}=1+\frac{1}{\pi} \int_{0}^{2 \pi} \int_{\Delta(T, \varphi)}^{\infty}\left(\frac{\partial f}{\partial E}\right) \frac{E d E d \varphi}{\sqrt{E^{2}-\Delta_{i}(T, \varphi)^{2}}},
$$

where $f=\left[1+\exp \left(E / k_{B} T\right)\right]^{-1}$ is the Fermi function, $\varphi$ is the angle along the Fermi surface, and $\Delta_{i}(T, \varphi)=$ $\Delta_{0, i} \Gamma\left(T / T_{c}\right) g(\varphi)\left(\Delta_{0, i}\right.$ is the maximum gap value at $T=$ $0)$. The temperature dependence of the gap is approximated by the expression $\Gamma\left(T / T_{c}\right)=\tanh \left\{1.82\left[1.018\left(T_{c} / T-\right.\right.\right.$ 1) $\left.]^{0.51}\right\}$ [42], while $g(\varphi)$ describes the angular dependence of the gap and it is replaced by 1 for an $s$-wave gap, $[1+a \cos (4 \varphi) /(1+a)]$ for an anisotropic $s$-wave gap, and $|\cos (2 \varphi)|$ for a $d$-wave gap [43].
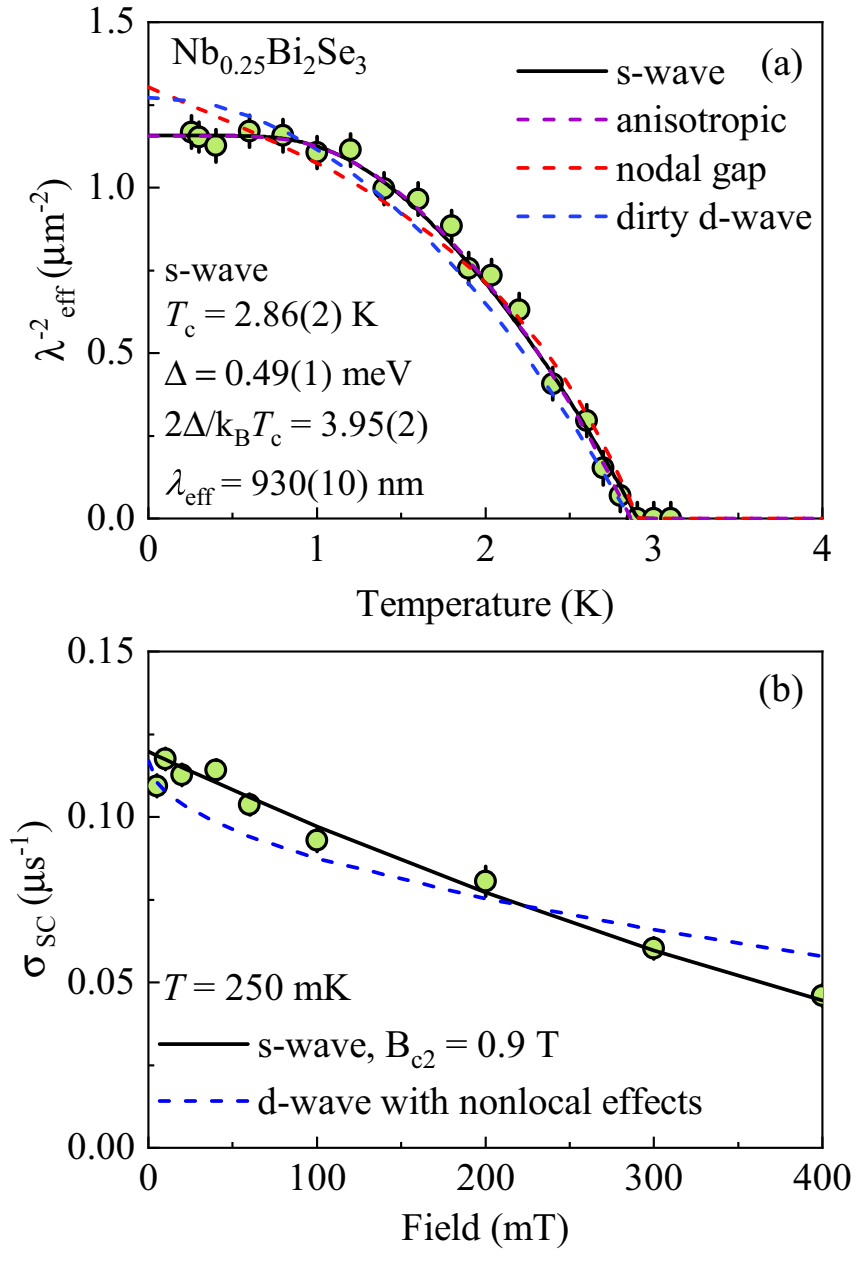

FIG. 4. Temperature and field evolution of $\lambda^{-2}$ for $\mathrm{Nb}_{0.25} \mathrm{Bi}_{2} \mathrm{Se}_{3}$. (a) The temperature dependence of $\lambda^{-2}$ for $\mathrm{Nb}_{0.25} \mathrm{Bi}_{2} \mathrm{Se}_{3}$, measured in an applied field of $\mu_{0} H=0.01 \mathrm{~T}$. The solid black line corresponds to a single-gap BCS $s$-wave model. The dashed purple line corresponds to anisotropic $s$-wave model. The dashed red line corresponds to clean-limit $d$-wave model. The dashed blue line corresponds to a dirty-limit $d$-wave model [a power law $1-\left(T / T_{c}\right)^{n}$ with $n=2$ ]. The error bars are calculated as the standard error of the mean. (b) Field dependence of the superconducting muon spin depolarization rate $\sigma_{\mathrm{sc}}$.

As seen in Fig. 4(a), the experimentally obtained $\lambda(T)$ dependence is best described by a momentum-independent $s$-wave model with a gap value of $\Delta=0.49(1) \mathrm{meV}$ and $T_{c}=2.86(2) \mathrm{K}$. We note that $T_{c}$ obtained from $\mu \mathrm{SR}$ is very close to those derived from susceptibility and specific-heat measurements [see Fig. 1(b)]. An anisotropic $s$-wave model, which would be consistent with the recently proposed fully gapped $\Delta_{4 y}$ case, where the superconducting gap has deep minima along the $k_{x}$ direction, describes the experimental data but only with small angular variation of the gap $[a=0.11(9)]$. We note that the $\left(p_{x}+\mathrm{i} p_{y}\right)$ pairing symmetry is also characterized by the full gap in $2 \mathrm{D}$ systems and would also give saturated behavior at low temperatures. However, the possibility of $p_{x}+i p_{y}$ pairing is excluded by the absence of the TRS-breaking state. Also $d$-wave gap symmetry was tested, but was found to be inconsistent with the data [for example, see the dashed line in Fig. 4(a)]. In particular, it is difficult to 
account for the very weak temperature dependence of $\lambda(T)$ at low $T$ within such models. We also tested the power law $\left[1-\left(\mathrm{T} / T_{c}\right)^{2}\right]$ which has been proposed theoretically [44] for the superfluid density of dirty $d$-wave superconductors and found it to be inconsistent with the data [see Fig. 4(a)]. This leaves a nodeless or fully gapped state as the most plausible bulk $\mathrm{SC}$ pairing state in the bulk of $\mathrm{Nb}_{0.25} \mathrm{Bi}_{2} \mathrm{Se}_{3}$. It should be noted, though, that saturation of the muon depolarization rate can arise under certain conditions even in nodal superconductors [45-49] and that in such cases extensions beyond Eq. (2) may be required to extract the superfluid density. In this particular case, a fully gapped state is substantiated by the field-dependent measurements. Figure 4(b) shows the obtained field dependence of $\sigma_{\mathrm{sc}}$ at $250 \mathrm{mK}$. Each point was obtained by field-cooling the sample from above $T_{c}$ to $250 \mathrm{mK}$. As expected from the London model one observes the maximum at $10 \mathrm{mT}$. Above $10 \mathrm{mT}, \sigma_{\mathrm{sc}}$ decreases with increasing magnetic field. This appears consistent with a behavior expected for the $s$-wave superconductor for an ideal triangular vortex lattice [39]. Taking into account the critical field of $0.9 \mathrm{~T}$, the theoretical $s$-wave behavior has been calculated according to the Brandt formula [39]. It is shown by the solid black line in Fig. 4(b), which agrees well with the experimental data. The model with nodes in the gap (dashed blue line), taking into account nonlocal effects [47], was also tested and found to be inconsistent with the data. Thus, the combination of temperature and field dependent measurements of $\sigma_{\mathrm{sc}}$ provides strong evidence for the fully gapped state. The ratio of the SC gap to $T_{c}$ was estimated to be $\left(2 \Delta / k_{B} T_{c}\right) \simeq 3.95$, which is consistent with the strongcoupling limit BCS expectation [16]. However, a similar ratio can also be expected for the Bose-Einstein condensation (BEC) like picture. It is important to note that the ratio $\Delta / k_{B} T_{c}$ does not effectively distinguish between BCS or BEC condensation. Having in mind $100 \%$ SC volume fraction, the effective penetration depth, $\lambda_{\text {eff }}$, at zero temperature is found to be $930(10) \mathrm{nm}$. Such a high value of $\lambda_{\text {eff }}$ was also estimated previously for other sister compounds, namely $\mathrm{Cu}$ and $\mathrm{Sr}$ doped $\mathrm{Bi}_{2} \mathrm{Si}_{3}[24,50,51]$. In view of the short coherence length and relatively large mean-free path $l \sim 50 \mathrm{~nm}$ [20], we can assume that $\mathrm{Nb}_{0.25} \mathrm{Bi}_{2} \mathrm{Se}_{3}$ lies close to the clean limit. With this assumption, we obtain the ground-state value of the carrier density $n_{s} /\left(m^{*} / m_{e}\right) \simeq 0.25 \times 10^{26} \mathrm{~m}^{-3}$. This density is by factor of $\sim 7$ smaller than the one $1.67 \times 10^{26} \mathrm{~m}^{-3}$ obtained for the unconventional Weyl superconductor $T_{d}-\mathrm{MoTe}_{2}$ [16] with the similar $T_{c}$. This fact indicates the relatively high $T_{c}$ for a small number of carriers and the $T_{c} / \lambda_{\text {eff }}^{-2}$ ratio is comparable to those of high-temperature unconventional superconductors $[52,53]$.

In order to support the conclusion derived from $\mu \mathrm{SR}$ investigation, we have performed an analysis of the superconducting part of the specific heat. The superconducting part of the specific heat $C_{\mathrm{el}}\left(=C_{S}-C_{N}\right)$ is related to the the entropy $S_{\mathrm{sc}}$ in the superconducting state by the relation $C_{\mathrm{el}}=T\left(\frac{\delta S_{\mathrm{sc}}}{\delta T}\right)$. Now, within the formulation of BCS theory, $S_{\mathrm{sc}}$ can be written as $[54,55]$

$$
S_{\mathrm{sc}}=-\frac{3 \gamma_{n}}{\kappa_{B} \pi^{3}} \int_{0}^{2 \pi} \int_{0}^{\infty}[(1-f) \ln (1-f)+f \ln f] d \varepsilon d \phi,
$$

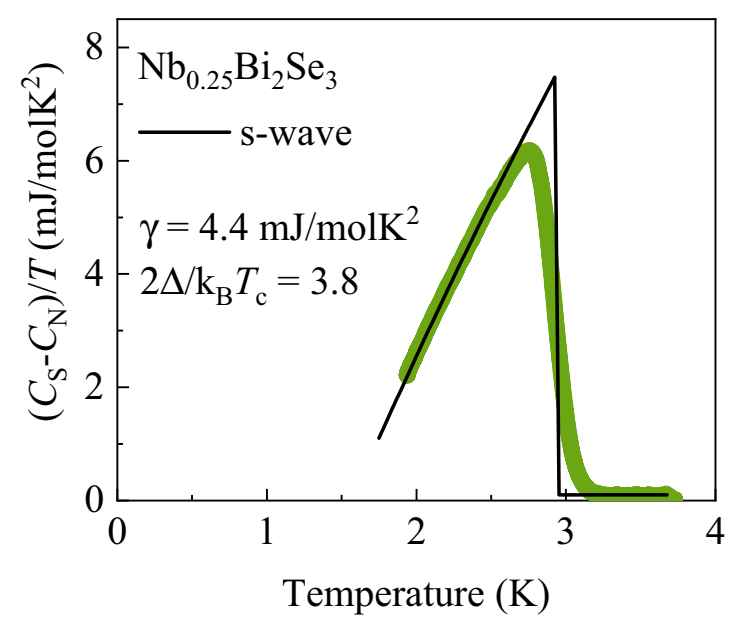

FIG. 5. Temperature evolution of specific heat for $\mathrm{Nb}_{0.25} \mathrm{Bi}_{2} \mathrm{Se}_{3}$. Temperature evolution of the electronic part of specific heat fitted with single $s$-wave gap model (solid line).

where $\gamma_{n}$ is the normal-state Sommerfeld coefficient, and $f$ is the Fermi function with energy $E=\sqrt{\varepsilon^{2}+\Delta^{2}(\phi, T)}$. Therefore, applying the same methodology as adapted for analyzing $\lambda(T)$, we can analyze the specific-heat data. Figure 5 depicts that, over the range of measured temperature, the superconducting contribution to the specific heat can also be modeled by a fully gapped $s$-wave picture, yielding $\Delta / k_{B} T_{c}=1.9$, consistent with the $\mu \mathrm{SR}$ measurements. However, this result has to be taken cautiously since we could not access the low-temperature region of the specific heat.

\section{DISCUSSION}

One of the findings of this paper is the absence of magnetism and time-reversal symmetry breaking state in the bulk of $\mathrm{Nb}_{0.25} \mathrm{Bi}_{2} \mathrm{Se}_{3}$ below $T_{c}$, classifying this system as a nonmagnetic and time-reversal invariant superconductor. This is in contrast with a previous report [29] which suggested a TRS-breaking state with triplet chiral $p$-wave pairing. Such conclusion was made based on the observation of anomalous Hall resistivity below $T_{c}$ and taking into account some theoretical aspects of impurity scattering in $p$-wave superconductors. The discrepancy between our results and the previous report might be related to the fact that the Hall response may contain surface effects, while with $\mu$ SR we are probing deep in the bulk. In addition, the anomalous Hall response within the SC state may originate not from magnetism but rather from an intrinsic topological band structure and Berry curvature effects.

In addition to the TRS-invariant SC state, another interesting observation is the fact that the $T_{c} / \lambda_{\text {eff }}^{-2}$ ratio for $\mathrm{Nb}_{0.25} \mathrm{Bi}_{2} \mathrm{Se}_{3}$ is comparable to those of high-temperature unconventional superconductors. Within the picture of BEC to BCS crossover [52,53], systems exhibiting small $T_{c} / \lambda_{\text {eff }}^{-2} \sim$ $0.00025-0.015$ are considered to be on the BCS-like side, while the large value of $T_{c} / \lambda_{\text {eff }}^{-2} \sim 1-20$ and the linear relationship between $T_{c}$ and $\lambda_{\text {eff }}^{-2}$ is expected only on the BEC-like side and is considered a hallmark feature of unconventional superconductivity. This approach has in the past been used 
for the characterization of BCS-like (so-called conventional) and BEC-like (so-called unconventional) superconductors. The presented results on $\mathrm{Nb}_{0.25} \mathrm{Bi}_{2} \mathrm{Se}_{3}$ demonstrate the ratio $T_{c} / \lambda_{\text {eff }}^{-2} \sim 2.5$, which is close to the one $T_{c} / \lambda_{\text {eff }}^{-2} \sim 4$ for hole-doped cuprates. This result provides strong evidence for an unconventional pairing mechanism in $\mathrm{Nb}_{0.25} \mathrm{Bi}_{2} \mathrm{Se}_{3}$, a system which exhibits the topologically nontrivial electronic structure. In addition, there are few other signatures of unconventional superconductivity in $\mathrm{Nb}_{0.25} \mathrm{Bi}_{2} \mathrm{Se}_{3}$ : namely, (1) similar to other unconventional superconductors, the multipleorbit nature of the electronic state [56] was observed in $\mathrm{Nb}_{0.25} \mathrm{Bi}_{2} \mathrm{Se}_{3}$, which might point to Fermi surface nesting as a possible superconducting mechanism; (2) the emergence of a pronounced twofold in-plane anisotropy of all superconducting properties and power-law temperature dependence of the penetration depth at the surface [21,22]; (3) unusual paramagnetic shift (this work); (4) a substantial increase in electron scattering by disorder is required to suppress $T_{c}$ in $\mathrm{Nb}_{0.25} \mathrm{Bi}_{2} \mathrm{Se}_{3}$, far larger than anticipated via conventional theory [22]; (5) $\mathrm{Nb}_{0.25} \mathrm{Bi}_{2} \mathrm{Se}_{3}$ manifests a very unusual upper critical field behavior signaling an unconventional nature of the superconducting state in this compound [23]. Similar to many unconventional superconductors, including the topological Weyl superconductor $T_{d}-\mathrm{MoTe}_{2}$ [16], we observed a fully gapped superconducting state in the bulk of $\mathrm{Nb}_{0.25} \mathrm{Bi}_{2} \mathrm{Se}_{3}$. The combination of unconventional superconductivity, fully gapped SC, and TRS-invariant state in the bulk of $\mathrm{Nb}_{0.25} \mathrm{Bi}_{2} \mathrm{Se}_{3}$ can be consistent with the particular class of topological superconducting phase belonging to the same symmetry as the Balian-Werthamer state [57], i.e., similar to the $B$ phase of ${ }^{3} \mathrm{He}$. Note that we observed only one SC gap in this multiband system $\mathrm{Nb}_{0.25} \mathrm{Bi}_{2} \mathrm{Se}_{3}$. ARPES and Hall effect measurements revealed the $n$-type conductivity in the normal state of $\mathrm{Nb}_{0.25} \mathrm{Bi}_{2} \mathrm{Se}_{3}$ and suggested that a single type of electron carrier is dominant with the normal-state carrier density $[29,56] n_{e} \simeq 1.5 \times 10^{26} \mathrm{~m}^{-3}$. The Hall carrier density is generally an order of magnitude larger than the carrier density given by the ellipsoidal Fermi surfaces. The carrier density of the ellipsoidal Fermi surfaces was reported [56] $n_{e} \simeq 2.6 \times 10^{25} \mathrm{~m}^{-3}$ for $\mathrm{Nb}_{0.25} \mathrm{Bi}_{2} \mathrm{Se}_{3}$. It is interesting that the SC carrier density $n_{s}$ (using $m^{*} / m_{e}=0.26$, the maximum value from Ref. [56]) $\simeq 0.7 \times 10^{25} \mathrm{~m}^{-3}$, which we estimated for $\mathrm{Nb}_{0.25} \mathrm{Bi}_{2} \mathrm{Se}_{3}$ is very close to the normal-state electron density, indicating that the superconductivity stems mostly from electron carriers. Thus, the single-gap superconductivity in $\mathrm{Nb}_{0.25} \mathrm{Bi}_{2} \mathrm{Se}_{3}$ may be explained by the superconducting gap occurring only on the electron-like Fermi surface.

Regarding the gap symmetry in $\mathrm{Nb}_{0.25} \mathrm{Bi}_{2} \mathrm{Se}_{3}$, using the tunnel-diode oscillator (TDO) technique Smylie et al. [21,22] showed that change in London penetration depth $\Delta \lambda(T)$ follows a power-law $\Delta \lambda(T) \sim T^{2}$ dependence indicating the presence of symmetry-protected point nodes. However, it has to be noted that TDO is a surface-sensitive technique whereas $\mu \mathrm{SR}$ is a bulk probe. More precisely, the response of a superconductor in the Meissner state to an AC electromagnetic field with frequency far below the gap frequency is described by the temperature-dependent anisotropic London penetration depth [58]. Therefore, in a TDO experiment, only material in the near-surface area on the order of a few $\lambda$ is probed while in a $\mu \mathrm{SR}$ experiment the whole sample volume is probed.
The $\mu \mathrm{SR}$ experiments presented here yield an upper limit of lambda of $\sim 900 \mathrm{~nm}$, while previous determinations based on measurements of the lower critical field yielded $\sim 250 \mathrm{~nm}$ for the in-plane penetration depth [21]. Typically, TDO measurements are interpreted as representing the bulk behavior of the superconductor. However, recent theoretical analysis by $\mathrm{Wu}$ et al. [59] suggests that the formation of a surface state at the surface of a topological superconductor causes an effective penetration depth describing the electromagnetic fields near the surface which contains a correction term with power-law temperature dependence. The authors considered the fully gapped SC state in the bulk. In addition to the bulk-dominated London response, they identified an additional $\Delta \lambda(T) \sim T^{3}$ power-law-in-temperature contribution from the surface, valid in the low-temperature limit, and it was argued that the powerlaw temperature dependence of the penetration depth can be one indicator of topological superconductivity. Since TDO probes the near-surface electromagnetic fields it will sense this correction and nonexponential temperature dependence while this will go completely unnoticed in $\mu \mathrm{SR}$ or specific heat. Currently, it is not clear why the large in-plane anisotropy, which is an established bulk effect in $\mathrm{Nb}_{0.25} \mathrm{Bi}_{2} \mathrm{Se}_{3}$, is not reflected in the bulk SC gap structure. Future work will be needed to address this important issue.

Finally, the observation of the paramagnetic shift in the $\mathrm{SC}$ state of $\mathrm{Nb}_{0.25} \mathrm{Bi}_{2} \mathrm{Se}_{3}$, instead of the expected diamagnetic shift imposed by the SC state, deserves some attention. In general, the paramagnetic shift can have few possible causes: field-induced magnetism [60,61], vortex disorder [62], oddfrequency superconducting state [63], and by the suppression of the negative Knight shift below $T_{c}$ due to singlet pairing. Considering the absence of any trace of magnetism in $\mathrm{Nb}_{0.25} \mathrm{Bi}_{2} \mathrm{Se}_{3}$ and the symmetric line shape from TF- $\mu \mathrm{SR}$, magnetism and disorder effects can be excluded as being the origin of the observed paramagnetic shift. The shape of the $\sigma_{\mathrm{sc}}(T)$ is also not consistent with odd-frequency superconducting pairing. Additional experiments are needed to explore the origin of such an unconventional paramagnetic shift in $\mathrm{Nb}_{0.25} \mathrm{Bi}_{2} \mathrm{Se}_{3}$.

\section{CONCLUSIONS}

In conclusion, we provide a microscopic investigation of the superconductivity in the layered topological superconductor candidate $\mathrm{Nb}_{0.25} \mathrm{Bi}_{2} \mathrm{Se}_{3}$ with a bulk probe. Specifically, the zero-temperature magnetic penetration depth $\lambda_{\text {eff }}(0)$ and the temperature as well as the field dependence of $\lambda_{\text {eff }}^{-2}$ were studied by means of $\mu$ SR experiments. The superfluid density can be described in a scenario of a complete gap. However, future work will explore the relation between the measured muon depolarization rate and the penetration depth in anisotropic and possibly inhomogeneous systems. Interestingly, the $T_{c} / \lambda_{\text {eff }}^{-2}$ ratio is comparable to those of high-temperature unconventional superconductors, pointing to the unconventional nature of superconductivity in $\mathrm{Nb}_{0.25} \mathrm{Bi}_{2} \mathrm{Se}_{3}$. Furthermore, $\mu \mathrm{SR}$, which is an extremely sensitive magnetic probe, does not show evidence of any spontaneous magnetic fields that would be expected for a TRS-breaking state in the bulk of $\mathrm{Nb}_{0.25} \mathrm{Bi}_{2} \mathrm{Se}_{3}$. Our results classify $\mathrm{Nb}_{0.25} \mathrm{Bi}_{2} \mathrm{Se}_{3}$ as an unconventional timereversal invariant and fully gapped bulk superconductor. 


\section{ACKNOWLEDGMENTS}

The work was performed at the Swiss Muon Source $(\mathrm{S} \mu \mathrm{S})$, Paul Scherrer Institute, Villigen, Switzerland. Work in Okayama is supported by JSPS KAKENHI Grants No. 18K03540 and No. 19H01852. M.Z.H. acknowledges visiting scientist support from IQIM at the California Institute of Technology. K.W. acknowledges funding from the SNSF through a postdoctoral mobility fellowship. Specific-heat measurements were supported by the US Department of Energy, Office of Science, Basic Energy Sciences, Materials Sciences and Engineering Division.
[1] L. V. Levitin, R. G. Bennett, A. Casey, B. Cowan, J. Saunders, D. Drung, Th. Schurig, and J. M. Parpia, Science 340, 841 (2013).

[2] X.-L. Qi and S.-C. Zhang, Rev. Mod. Phys. 83, 1057 (2011).

[3] Y. Ando and L. Fu, Annu. Rev. Condens. Matter Phys. 6, 361 (2015).

[4] M. Sato and Y. Ando, Rep. Prog. Phys. 80, 076501 (2017).

[5] M. Z. Hasan and C. L. Kane, Rev. Mod. Phys. 82, 3045 (2010).

[6] S.-Y. Xu, N. Alidoust, I. Belopolski, A. Richardella, C. Liu, M. Neupane, G. Bian, S.-H. Huang, R. Sankar, C. Fang, B. Dellabetta, W. Dai, Q. Li, M. J. Gilbert, F. Chou, N. Samarth, and M. Z. Hasan, Nat. Phys. 10, 943 (2014).

[7] A. Y. Kitaev, Phys. Usp. 44, 131 (2001).

[8] F. Wilczek, Nat. Phys. 5, 614 (2009).

[9] C. W. J. Beenakker, Annu. Rev. Condens. Matter Phys. 4, 113 (2013).

[10] M. Novak, S. Sasaki, M. Kriener, K. Segawa, and Y. Ando, Phys. Rev. B 88, 140502(R) (2013).

[11] S. Sasaki and T. Mizushima, Physica C 514, 206 (2015).

[12] K. Matano, M. Kriener, K. Segawa, Y. Ando, and G. Zheng, Nat. Phys. 12, 852 (2016).

[13] R. D. Zhong, J. A. Schneeloch, X. Y. Shi, Z. J. Xu, C. Zhang, J. M. Tranquada, Q. Li, and G. D. Gu, Phys. Rev. B 88, 020505(R) (2013).

[14] L. Fu and E. Berg, Phys. Rev. Lett. 105, 097001 (2010).

[15] G. M. Luke, Y. Fudamoto, K. M. Kojima, M. I. Larkin, J. Merrin, B. Nachumi, Y. J. Uemura, Y. Maeno, Z. Q. Mao, Y. Mori, H. Nakamura, and M. Sigrist, Nature (London) 394, 559 (1998).

[16] Z. Guguchia, F. von Rohr, Z. Shermadini, A. T. Lee, S. Banerjee, A. R. Wieteska, C. A. Marianetti, B. A. Frandsen, H. Luetkens, Z. Gong, S. C. Cheung, C. Baines, A. Shengelaya, G. Taniashvili, A. N. Pasupathy, E. Morenzoni, S. J. L. Billinge, A. Amato, R. J. Cava, R. Khasanov, and Y. J. Uemura, Nat. Commun. 8, 1082 (2017).

[17] Y. S. Hor, A. J. Williams, J. G. Checkelsky, P. Roushan, J. Seo, Q. Xu, H. W. Zandbergen, A. Yazdani, N. P. Ong, and R. J. Cava, Phys. Rev. Lett. 104, 057001 (2010).

[18] M. Kriener, K. Segawa, Z. Ren, S. Sasaki, and Y. Ando, Phys. Rev. Lett. 106, 127004 (2011).

[19] Z. Liu, X. Yao, J. Shao, M. Zuo, L. Pi, S. Tan, C. Zhang, and Y. Zhang, J. Am. Chem. Soc. 137, 10512 (2015).

[20] M. P. Smylie, K. Willa, K. Ryan, H. Claus, W.-K. Kwok, Y. Qiu, Y. S. Hor, and U. Welp, Physica C 543, 58 (2017).

[21] M. P. Smylie, H. Claus, U. Welp, W.-K. Kwok, Y. Qiu, Y. S. Hor, and A. Snezhko, Phys. Rev. B 94, 180510(R) (2016).

[22] M. P. Smylie, K. Willa, H. Claus, A. Snezhko, I. Martin, W.-K. Kwok, Y. Qiu, Y. S. Hor, E. Bokari, P. Niraula, A. Kayani, V. Mishra, and U. Welp, Phys. Rev. B 96, 115145 (2017).
[23] K. Kobayashi, T. Ueno, H. Fujiwara, T. Yokoya, and J. Akimitsu, Phys. Rev. B 95, 180503(R) (2017).

[24] J. A. Krieger, A. Kanigel, A. Ribak, E. Pomjakushina, K. B. Chashka, K. Conder, E. Morenzoni, T. Prokscha, A. Suter, and Z. Salman, JPS Conf. Proc. 21, 011028 (2018).

[25] L. Fu, Phys. Rev. B 90, 100509(R) (2014).

[26] S. Yonezawa, Condens. Matter 4, 2 (2019).

[27] J. W. F. Venderbos, V. Kozii, and L. Fu, Phys. Rev. B 94, 180504(R) (2016).

[28] Y. Nagai, H. Nakamura, and M. Machida, Phys. Rev. B 86, 094507 (2012).

[29] Y. Qiu, K. N. Sanders, J. Dai, J. E. Medvedeva, W. Wu, P. Ghaemi, T. Vojta, and Y. S. Hor, arXiv:1512.03519.

[30] C. Kurter, A. Finck, E. Huemiller, J. Medvedeva, A. Weis, J. Atkinson, Y. Qiu, L. Shen, S. H. Lee, T. Vojta, P. Ghaemi, Y. S. Hor, and D. V. Harlingen, Nano Lett. 19, 38 (2019).

[31] J. Shen, W. Y. He, N. F. Q. Yuan, Z. Huang, C. W. Cho, S. H. Lee, Y. S. Hor, K. T. Law, and R. Lortz, npj Quantum Mater. 2, 59 (2017).

[32] T. Asaba, B. J. Lawson, C. Tinsman, L. Chen, P. Corbae, G. Li, Y. Qiu, Y. S. Hor, L. Fu, and L. Li, Phys. Rev. X 7, 011009 (2017).

[33] K. Willa, Z. Diao, D. Campanini, U. Welp, R. Divan, M. Hudl, Z. Islam, W.-K. Kwok, and A. Rydh, Rev. Sci. Instrum. 88, 125108 (2017).

[34] S. Tagliati, V. M. Krasnov, and A. Rydh, Rev. Sci. Instrum. 83, 055107 (2012).

[35] K. Willa, R. Willa, K. W. Song, G. D. Gu, J. A. Schneeloch, R. Zhong, A. E. Koshelev, W.-K. Kwok, and U. Welp, Phys. Rev. B 98, 184509 (2018).

[36] A. Suter and B. Wojek, Phys. Procedia 30, 69 (2012).

[37] R. Kubo and T. Toyabe, in Magnetic Resonance and Relaxation, edited by R. Blinc (North-Holland, Amsterdam, 1967), p. 810; T. Toyabe, M.S. thesis, University of Tokyo, 1966.

[38] A. D. Hillier, Q. Jorge, and R. Cywinski, Phys. Rev. Lett. 102, 117007 (2009).

[39] E. H. Brandt, Phys. Rev. B 37, 2349 (1988).

[40] E. H. Brandt, Phys. Rev. B 68, 054506 (2003).

[41] M. Tinkham, Introduction to Superconductivity (Krieger Publishing Company, Malabar, FL, 1975).

[42] A. Carrington and F. Manzano, Physica C 385, 205 (2003).

[43] M. H. Fang, H. M. Pham, B. Qian, T. J. Liu, E. K. Vehstedt, Y. Liu, L. Spinu, and Z. Q. Mao, Phys. Rev. B 78, 224503 (2008).

[44] P. J. Hirschfeld, W. O. Putikka, and D. J. Scalapino, Phys. Rev. B 50, 10250 (1994).

[45] J. E. Sonier, J. H. Brewer, and R. F. Kiefl, Rev. Mod. Phys. 72, 769 (2000).

[46] R. Khasanov, A. Shengelaya, K. Conder, E. Morenzoni, I. M. Savic, J. Karpinski, and H. Keller, Phys. Rev. B 74, 064504 (2006). 
[47] H. Luetkens, H.-H. Klauss, R. Khasanov, A. Amato, R. Klingeler, I. Hellmann, N. Leps, A. Kondrat, C. Hess, A. Kohler, G. Behr, J. Werner, and B. Buchner, Phys. Rev. Lett. 101, 097009 (2008).

[48] J. E. Sonier, J. Phys. Soc. Jpn. 85, 091005 (2016).

[49] D. R. Harshman and A. T. Fiory, J. Phys.: Condens. Matter 23, 315702 (2011).

[50] H. Leng, D. Cherian, Y. K. Huang, J.-C. Orain, A. Amato, and A. de Visser, Phys. Rev. B 97, 054503 (2018).

[51] P. Neha, P. K. Biswas, T. Das, and S. Patnaik, Phys. Rev. Mater. 3, 074201 (2019).

[52] Y. J. Uemura, G. M. Luke, B. J. Sternlieb, J. H. Brewer, J. F. Carolan, W. N. Hardy, R. Kadono, J. R. Kempton, R. F. Kiefl, S. R. Kreitzman, P. Mulhern, T. M. Riseman, D. L. Williams, B. X. Yang, S. Uchida, H. Takagi, J. Gopalakrishnan, A. W. Sleight, M. A. Subramanian, C. L. Chien, M. Z. Cieplak, G. Xiao, V. Y. Lee, B. W. Statt, C. E. Stronach, W. J. Kossler, and X. H. Yu, Phys. Rev. Lett. 62, 2317 (1989).

[53] Y. J. Uemura et al., Phys. Rev. Lett. 66, 2665 (1991).

[54] Y. Nakajima, T. Nakagawa, T. Tamegai, and H. Harima, Phys. Rev. Lett. 100, 157001 (2008).
[55] J. Chen, L. Jiao, J. L. Zhang, Y. Chen, L. Yang, M. Nicklas, F. Steglich, and H. Q. Yuan, New J. Phys. 15, 053005 (2013).

[56] B. J. Lawson et al., Phys. Rev. B 94, 041114(R) (2016).

[57] R. Balian and N. R. Werthamer, Phys. Rev. 131, 1553 (1963).

[58] R. Prozorov and R. W. Giannetta, Supercond. Sci. Technol. 19, R41 (2006).

[59] T. C. Wu, H. K. Pal, P. Hosur, and M. S. Foster, Phys. Rev. Lett. 124, 067001 (2020).

[60] R. Khasanov, A. Maisuradze, H. Maeter, A. Kwadrin, H. Luetkens, A. Amato, W. Schnelle, H. Rosner, A. Leithe-Jasper, and H.-H. Klauss, Phys. Rev. Lett. 103, 067010 (2009).

[61] Z. Guguchia, R. Khasanov, Z. Bukowski, F. von Rohr, M. Medarde, P. K. Biswas, H. Luetkens, A. Amato, and E. Morenzoni, Phys. Rev. B 93, 094513 (2016).

[62] J. E. Sonier, W. Huang, C. V. Kaiser, C. Cochrane, V. Pacradouni, S. A. Sabok-Sayr, M. D. Lumsden, B. C. Sales, M. A. McGuire, A. S. Sefat, and D. Mandrus, Phys. Rev. Lett. 106, 127002 (2011).

[63] J. A. Krieger, A. Pertsova, S. R. Giblin, M. Dobeli, T. Prokscha, C. W. Schneider, A. Suter, T. Hesjedal, A. V. Balatsky, and Z. Salman, Phys. Rev. Lett. 125, 026802 (2020). 